\title{
Stability of Time-delay System with Time-varying Uncertainties via Homogeneous Polynomial Lyapunov-Krasovskii Functions
}

\author{
Guo-Chen Pang Kan-Jian Zhang \\ Key Laboratory of Measurement and Control of CSE Ministry of Education, School of Automation, Southeast University, \\ Nanjing 210096, China
}

\begin{abstract}
This paper deals with the robust stability of time-delay system with time-varying uncertainties via homogeneous polynomial Lyapunov-Krasovskii functions (HPLKF). We give a sufficient condition to demonstrate that the system is asymptotically stable. A new class of Lyapunov-Krasovskii function is introduced, whose main feature is that the conservativeness due to uncertainties is reduced. Numerical examples illustrate the effectiveness of our method.
\end{abstract}

Keywords: Asymptotically stable, time-delay, uncertainties, linear matrix inequality (LMI), homogeneous polynomial.

\section{Introduction}

Systems with unknown delayed states are often encountered in practice, such as communication systems, engineering systems and process control systems, etc.. For this reason, robust stability analysis for uncertain time-delay systems has attracted a considerable amount of interests in recent years ${ }^{[1-12]}$. The quadratic Lyapunov-Krasovskii (L$\mathrm{K}$ ) function is always employed and the results are often obtained in the form of linear matrix inequalities (LMIs). However this method gives rise to overly conservativeness. So, many researchers reduce the conservativeness due to uncertainties through changing the structure of the quadratic Lyapunov-Krasovskii function ${ }^{[1]}$. In [2], some new robust stability criteria for linear sytems with interval time-varying delay have been investigated. This paper is based around using homogeneous polynomial Lyapunov-Krasovskii functions (HPLKF) to study the conservativeness due to uncertainties. By using this method, the conservativeness due to uncertainties is reduced.

Recently, a more general class of Lyapunov functions are named homogeneous polynomial Lyapunov functions $(\mathrm{HPLF})^{[13-24]}$. The robustness analysis via homogeneous polynomial Lyapunov functions has been presented in many papers. In [13], the authors demonstrated simultaneous stability by using non-quadratic polynomial Lyapunov functions. Whereas $\mathrm{Xu}$ and $\mathrm{Xie}^{[14]} \mathrm{ad}-$ dressed the construction of piecewise homogeneous polynomial Lyapunov functions for piecewise affine systems.

\footnotetext{
Regular paper

Manuscript received on January 27, 2014; accepted on May 30, 2014 This project was supported by Major Programme of National Natural Science Foundation of China (No.11190015), National Natural Science Foundation of China (No.61374006) and Graduate Student Innovation Foundation of Jiangsu Province (No. 3208004904).

Recommended by Associate Editor Qing-Long Han

(C) Institute of Automation, Chinese Academy of Science and Springer-Verlag Berlin Heidelberg 2015
}

Asymptotic stability regions of nonlinear dynamical systems were estimated by using homogeneous polynomial Lyapunov functions $^{[15]}$. In [16], an LMI approach was given by homogeneous optimized forms.

In this paper, inspired by HPLF, we apply homogeneous polynomial Lyapunov Krasovskii functions for the timedelay system with time-varying polytopic type uncertainties. First of all, we give $h$ and $\mu$. Since the solution of system is continuous function of $p(t)$, there must exists an upper bound $k$, such that system is stable for all $p(t) \in[0, k]$. By solving a numerical example, we can get that the homogeneous polynomial Lyapunov-Krasovskii functions in this paper produce much better results than those from quadratic Lyapunov-Krasovskii functions.

The paper is organized as follows. In Section 2, we state the problem and some preliminaries on homogeneous polynomial functions. In Section 3, we present the sufficient condition of asymptotic stability of system. Section 4 illustrates the obtained result by numerical example, which is followed by the conclusion in Section 5 .

Notations. $\mathbf{R}^{n}$ denotes the $n$-dimension Euclidean space and $\mathbf{R}^{n \times m}$ is the set of real matrices with dimension $n \times m$; The notation $X \geq Y$ (respectively $X>Y$ ) where $X$ and $Y$ are symmetric matrices, means that the matrix $X-Y$ is positive semi-definite (respectively, positive definite); $A^{\mathrm{T}}$ denotes the transposed matrix of $A ; x \otimes y$ denotes the Kronecker product of vectors $x$ and $y$, i.e., $x \otimes y=\left(\begin{array}{l}x_{1} y \\ x_{2} y\end{array}\right), x=\left(\begin{array}{l}x_{1} \\ x_{2}\end{array}\right)$. 


\section{Problem statement and preliminaries}

A system containing time-varying structured uncertainties is described by

$$
\left\{\begin{array}{l}
\dot{x}(t)=A(p(t)) x(t)+A_{d}(p(t)) x_{d}, t>0 \\
x(t)=\phi(t), t \in[-h, 0]
\end{array}\right.
$$

where $x \in \mathbf{R}^{n}$ is the state, $x_{d}=x(t-d(t))$ stands for the delayed state, and $p(t) \in[0, k]$ is a time-varying uncertain parameter.

The matrices $A(p(t))$ and $A_{d}(p(t))$ of system contain uncertainties and satisfy the real, convex, polytopic-type model

$$
\begin{aligned}
{\left[A\left(p(t), A_{d}(p(t))\right)\right] } & =\sum_{1}^{n} \lambda_{i}(t)\left[A_{i}, A_{d i}\right] \\
\sum_{1}^{n} \lambda_{i}(t) & =1, \lambda_{i}(t) \geq 0 .
\end{aligned}
$$

The delay, $d(t)$, is a time-varying continuous function which satisfies

$$
0 \leq d(t) \leq h
$$

and

$$
\dot{d}(t) \leq \mu
$$

where $\tau$ and $\mu$ are positive scalars.

In this paper, we want to get the upper bound $k$. If $p(t) \in$ $[0, k]$, the system is asymptotically stable. For this purpose, we employ the Lyapunov-Krasovskii functions. Then, the problem reduces to an optimization problem.

However, because the quadratic Lyapunov-Krasovskii functions give fairly conservative result in general. In order to reduce the conservativeness, we employ homogeneous polynomial Lyapunov-Krasovskii functions. Our objective is to get a better result by using this method.

We firstly recall the homogeneous polynomial function.

Definition $\mathbf{1}^{[13]}$. A monomial is a product of variables raised to non-negative integer powers. For example

$$
x^{2} \text { and } x^{3} y z^{10}
$$

are both monomials.

Remark 1. The degree of a monomial is the sum of the integer powers. For example, the degree of $x y$ is 2 , and the degree of $x^{3}$ is 3 .

Definition $\mathbf{2}^{[14]}$. Consider the vector $x \in \mathbf{R}^{n}, x=$ $\left[x_{1}, \cdots, x_{n}\right]^{\mathrm{T}}$. The power transformation of degree $m$ is a nonlinear change of coordinates that forms a new vector $x^{m}$ of all integer powered monomials of degree $m$ that can be made from the original $x$ vector

$$
\begin{aligned}
& x_{j}^{m}=c_{j} x_{1}^{m_{j 1}} x_{2}^{m_{j 2}} \cdots x_{n}^{m_{j n}}, \quad m_{j i} \in \mathbf{N}^{+} \\
& \sum_{i=1}^{n} m_{j i}=m, j=1, \cdots, d_{n, m}, \quad d_{n, m}=\frac{(n+m-1) !}{(n-1) ! m !} .
\end{aligned}
$$

Usually we take $c_{l}=1$. For example

1) $n=2, m=3, \Longrightarrow d_{n, m}=4$.

$$
x=\left(\begin{array}{c}
x_{1} \\
x_{2}
\end{array}\right) \Longrightarrow x^{3}=\left(\begin{array}{c}
x_{1}^{3} \\
x_{1}^{2} x_{2} \\
x_{1} x_{2}^{2} \\
x_{2}^{3}
\end{array}\right) .
$$

2) $n=3, m=2, \Longrightarrow d_{n, m}=6$.

$$
x=\left(\begin{array}{l}
x_{1} \\
x_{2} \\
x_{3}
\end{array}\right) \Longrightarrow x^{2}=\left(\begin{array}{c}
x_{1}^{2} \\
x_{1} x_{2} \\
x_{1} x_{3} \\
x_{2}^{2} \\
x_{2} x_{3} \\
x_{3}^{2}
\end{array}\right) .
$$

Definition $3^{[14]}$. Let $P_{n, m}$ be the set of all polynomials in $n$ variables with degree $m$. If a polynomial $f_{n, m} \in P_{n, m}$ and $f_{n, m}(\lambda x)=\lambda^{m} f_{n, m}(x)$, then $f_{n, m}$ is a homogeneous polynomial. We define the set of all homogeneous polynomials in $n$ variables with degree $m$ as $H_{n, m}$.

Lemma $\mathbf{1}^{[15]}$. Let $H_{n, m}$ be the set of all homogeneous polynomials in $n$ variables with degree $m$. Then, $f(x) \in$ $H_{n, 2 m}$ if and only if there exists a non-zero matrix $Q_{0} \in$ $\mathbf{R}^{s \times s}, Q_{0}=Q_{0}^{\mathrm{T}}$ such that

$$
f(x)=x^{m \mathrm{~T}} Q_{0} x^{m} .
$$

Remark 2. $Q_{0}$ is not unique in the above representation of $f(x)$.

Definition $4^{[17]}$. Let us consider the system

$$
\dot{x}(t)=A x(t)
$$

where $x(t) \in \mathbf{R}^{n}$ and $A \in \mathbf{R}^{n \times n}$. Let $m \geq 1$ be an integer. Denote $\bar{A} \in \mathbf{R}^{\frac{(n+m-1) !}{(n-1) ! m !} \times \frac{(n+m-1) !}{(n-1) ! m !}}$ by the matrix satisfying the relation

$$
\frac{\mathrm{d}}{\mathrm{d} t} x^{m}(t)=\frac{\partial x^{m}}{\partial x} A x(t)=\bar{A} x^{m}(t) .
$$

The matrix $\bar{A}$ is called extended matrix of $A$.

Lemma 2 (Schur complement ${ }^{[3]}$ ). The linear matrix inequality (LMI)

$$
\left(\begin{array}{cc}
\Lambda_{1}(x) & \Lambda_{2}(x) \\
* & \Lambda_{3}(x)
\end{array}\right)>0
$$

is equivalent to $\Lambda_{1}(x)-\Lambda_{2}(x) \Lambda_{3}^{-1} \Lambda_{2}^{\mathrm{T}}(x)>0$ and $\Lambda_{3}(x)>0$, where $\Lambda_{1}(x)=\Lambda_{1}^{\mathrm{T}}(x), \Lambda_{3}(x)=\Lambda_{3}^{\mathrm{T}}(x)$ and $\Lambda_{2}(x)$ depends affinely on $x$

\section{Main results}

Let us introduce the extended system corresponding to (1) defined by

$$
\begin{aligned}
\frac{\mathrm{d}}{\mathrm{d} t} x^{m}(t)= & \frac{\partial x^{m}}{x} \sum_{i=1}^{n} \lambda_{i}(t)\left(A_{i} x(t)+A_{d i} x_{d}\right)= \\
& \sum_{i=1}^{n} \lambda_{i}(t)\left(\bar{A}_{i} x^{m}(t)+\bar{A}_{d i} T\left(x^{m-1}(t), x_{d}\right)\right)
\end{aligned}
$$

where $T\left(x^{m-1}(t), x_{d}(t)\right)$ stands for $x^{m-1}(t) \otimes x_{d}(m \geq 1)$. $T\left(x(t), x_{d}\right)=x(t) \otimes x_{d}=\left(\begin{array}{l}x_{1}(t) x_{1 d} \\ x_{1}(t) x_{2 d} \\ x_{2}(t) x_{1 d} \\ x_{2}(t) x_{2 d}\end{array}\right)$, where $x(t)=$ 
$\left(\begin{array}{l}x_{1}(t) \\ x_{2}(t)\end{array}\right)$

When $m=1, T\left(x^{m-1}(t), x_{d}\right)=x_{d}$.

Theorem 1. For given scalars $h>0$ and $\mu<1$ and a time-varying delay satisfying (3) and (4), the uncertainties satisfy $0 \leq p(t) \leq k$. If there exist $P=P^{\mathrm{T}} \geq 0, Q=Q^{\mathrm{T}} \geq$ $0, Z=Z^{\mathrm{T}}>0$, and matrices $X=\left(\begin{array}{ll}X_{11} & X_{12} \\ X_{21} & X_{22}\end{array}\right) \geq 0$, $N_{j}, j=1,2$ with proper dimensions such that

$$
\left(\begin{array}{ccc}
\Phi_{11} & P \bar{A}_{d i}-N_{1}+E^{\mathrm{T}} N_{2}^{\mathrm{T}}+h X_{12} & h F_{i}^{\mathrm{T}} Z \\
* & -(1-\mu) Q-N_{2}-N_{2}^{\mathrm{T}}+h X_{22} & h \bar{F}_{i}^{\mathrm{T}} Z \\
* & * & -h Z
\end{array}\right)=\Phi<0
$$$$
\left(\begin{array}{ccc}
X_{11} & X_{12} & N_{1} \\
* & X_{22} & N_{2} \\
* & * & Z
\end{array}\right)=\Psi \geq 0
$$

where

$$
\begin{gathered}
\Phi_{11}=\bar{A}_{i}^{\mathrm{T}} P+P \bar{A}_{i}+E^{\mathrm{T}} Q E+N_{1} E+ \\
E^{\mathrm{T}} N_{1}^{\mathrm{T}}+h X_{11}, \quad i=1, \cdots, n
\end{gathered}
$$

then the system (1) is asymptotically stable.

Proof. Choose the Lyapunov-Krasovskii functions candidate to be

$$
V\left(x_{t}^{2 m}\right)=V_{1}\left(x_{t}^{2 m}\right)+V_{2}\left(x_{t}^{2 m}\right)+V_{3}\left(x_{t}^{2 m}\right)
$$

where

$$
\begin{aligned}
& V_{1}\left(x_{t}^{2 m}\right)=x^{m \mathrm{~T}}(t) P x^{m}(t) \\
& V_{2}\left(x_{t}^{2 m}\right)= \\
& \int_{t-d(t)}^{t} T^{\mathrm{T}}\left(x^{m-1}(t), x(s)\right) Q T\left(x^{m-1}(t), x(s)\right) \mathrm{d} s \\
& V_{3}\left(x_{t}^{2 m}\right)= \\
& \int_{-h}^{0} \int_{t+\theta}^{t} T^{\mathrm{T}}\left(x^{m-1}(t), \dot{x}(s)\right) Z T\left(x^{m-1}(t), \dot{x}(s)\right) \mathrm{d} s \mathrm{~d} \theta .
\end{aligned}
$$

Consider the HPLKF given by (15). First, we have

$$
\varepsilon_{1}\left\|x_{t}^{m}(0)\right\| \leq V\left(x_{t}^{2 m}\right) \leq \varepsilon_{2}\left\|x_{t}^{m}\right\|
$$

where $\varepsilon_{1}=\lambda_{\min }(P), \varepsilon_{2}=\lambda_{\max }(P)+\mu \lambda_{\max }\left(E^{\mathrm{T}} Q E\right)+$ $h \lambda_{\max }\left(E^{\mathrm{T}} Z E\right)$.

For any matrix

$$
X=\left(\begin{array}{ll}
X_{11} & X_{12} \\
X_{21} & X_{22}
\end{array}\right) \geq 0
$$

the following inequality is true

$$
h \eta_{1}^{\mathrm{T}}(t) X \eta_{1}(t)-\int_{t-d(t)}^{t} \eta_{1}^{\mathrm{T}}(t) X \eta_{1}(t) \mathrm{d} s \geq 0
$$

where $\eta_{1}^{\mathrm{T}}(t)=\left[x^{m \mathrm{~T}}(t), T^{\mathrm{T}}\left(x^{m-1}(t), x_{d}\right)\right]$.
According to the Newton-Leibniz formula, we have

$$
\begin{array}{r}
2\left[x^{m \mathrm{~T}}(t) N_{1}+T^{\mathrm{T}}\left(x^{m-1}(t), x_{d}\right) N_{2}\right]\left[T\left(x^{m-1}(t), x(t)\right)-\right. \\
\left.\int_{t-d(t)}^{t} T\left(x^{m-1}(t), \dot{x}(s)\right) \mathrm{d} s-T\left(x^{m-1}(t), x_{d}\right)\right]=0 .
\end{array}
$$

Calculating the derivative $V_{1}\left(x_{t}^{2 m}\right)$ along the solutions of (12), we should get

$$
\begin{aligned}
& \frac{\mathrm{d}}{\mathrm{d} t} x^{m \mathrm{~T}}(t) P x^{m}(t)= \\
& \quad \sum_{i=1}^{n} \lambda_{i}(t)\left[\left(x^{m \mathrm{~T}}(t) \bar{A}_{i}^{\mathrm{T}}+T^{\mathrm{T}}\left(x^{m-1}(t), x_{d}\right) \bar{A}_{d i}^{\mathrm{T}}\right) P x^{m}(t)+\right. \\
& \left.\quad x^{m \mathrm{~T}}(t) P\left(\bar{A}_{i} x^{m}(t)+\bar{A}_{d i} T\left(x^{m-1}(t), x_{d}\right)\right)\right]= \\
& \quad \sum_{i=1}^{n} \lambda_{i}(t)\left[x^{m \mathrm{~T}}(t) \bar{A}_{i}^{\mathrm{T}} P x^{m}(t)+\right. \\
& \quad T^{\mathrm{T}}\left(x^{m-1}(t), x_{d}\right) \bar{A}_{d i}^{\mathrm{T}} P x^{m}(t)+ \\
& \quad x^{m \mathrm{~T}}(t) P \bar{A}_{i} x^{m}(t)+ \\
& \left.\quad x^{m \mathrm{~T}}(t) P \bar{A}_{d i} T\left(x^{m-1}(t), x_{d}\right)\right] .
\end{aligned}
$$

Similar to the derivative $V_{1}\left(x_{t}^{2 m}\right)$, the derivative $V_{2}\left(x_{t}^{2 m}\right)$ is

$$
\begin{aligned}
\frac{\mathrm{d}}{\mathrm{d} t} \int_{t-d(t)}^{t} T^{\mathrm{T}}\left(x^{m-1}(t), x(s)\right) Q T\left(x^{m-1}(t), x(s)\right) \mathrm{d} s= \\
\\
T^{\mathrm{T}}\left(x^{m-1}(t), x(t)\right) Q T\left(x^{m-1}(t), x(t)\right)- \\
\quad(1-\dot{d}(t)) T^{\mathrm{T}}\left(x^{m-1}(t), x_{d}\right) Q T\left(x^{m-1}(t), x_{d}\right) .
\end{aligned}
$$

Defining $T\left(x^{m-1}(t), x(t)\right)=E x^{m}$, so

$$
T^{\mathrm{T}}\left(x^{m-1}(t), x(t)\right) Q T\left(x^{m-1}(t), x(t)\right)=x^{m \mathrm{~T}}(t) E^{\mathrm{T}} Q E x^{m}(t) .
$$

The derivative $V_{3}\left(x_{t}^{2 m}\right)$ along the solutions of (12) is

$$
\begin{gathered}
\frac{\mathrm{d}}{\mathrm{d} t} \int_{-h}^{0} \int_{t+\theta}^{t} T^{\mathrm{T}}\left(x^{m-1}(t), \dot{x}(s)\right) Z T\left(x^{m-1}(t), \dot{x}(s)\right) \mathrm{d} s \mathrm{~d} \theta= \\
\quad h T^{\mathrm{T}}\left(x^{m-1}(t), \dot{x}(t)\right) Z T\left(x^{m-1}(t), \dot{x}(t)\right)- \\
\quad \int_{t-h}^{t} T^{\mathrm{T}}\left(x^{m-1}(t), \dot{x}(s)\right) Z T\left(x^{m-1}(t), \dot{x}(s)\right) \mathrm{d} s .
\end{gathered}
$$

Let $T\left(x^{m-1}(t), \dot{x}(t)\right)=\sum_{i=1}^{n} \lambda_{i}(t)\left[F_{i} x^{m}(t)+\right.$ $\left.\bar{F}_{i} T\left(x^{m-1}(t), x_{d}\right)\right]$. 
Thus,

$$
\begin{aligned}
& h T^{\mathrm{T}}\left(x^{m-1}(t), \dot{x}(t)\right) Z T\left(x^{m-1}(t), \dot{x}(t)\right)= \\
& \sum_{i=1}^{n} \lambda_{i}(t) h x^{m \mathrm{~T}}(t) F_{i}^{\mathrm{T}} Z \sum_{i=1}^{n} \lambda_{i}(t) F_{i} x^{m}(t)+ \\
& \sum_{i=1}^{n} \lambda_{i}(t) h x^{m \mathrm{~T}}(t) F_{i}^{\mathrm{T}} Z \sum_{i=1}^{n} \lambda_{i}(t) \bar{F}_{i} T\left(x^{m-1}(t), x_{d}\right)+ \\
& \quad \sum_{i=1}^{n} \lambda_{i}(t) h T^{\mathrm{T}}\left(x^{m-1}(t), x_{d}\right) \bar{F}_{i} Z \sum_{i=1}^{n} \lambda_{i}(t) F_{i} x^{m}(t)+ \\
& \sum_{i=1}^{n} \lambda_{i}(t) h T^{\mathrm{T}}\left(x^{m-1}(t), x_{d}\right) \bar{F}_{i} Z \\
& \sum_{i=1}^{n} \lambda_{i}(t) \bar{F}_{i} T\left(x^{m-1}(t), x_{d}\right) .
\end{aligned}
$$

Combining (19)-(25) and using the Schur complement, we have

$$
\dot{V}\left(x_{t}^{2 m}\right) \leq \sum_{i=1}^{n} \lambda_{i}(t)\left\{\eta_{1}^{\mathrm{T}}(t) \Phi \eta_{1}(t)-\int_{t-d(t)}^{t} \eta_{2}^{\mathrm{T}}(t) \Psi \eta_{2}(t) \mathrm{d} s\right\} .
$$

If $\Phi<0$ and $\Psi \geq 0$, then $\dot{V}^{2 m}\left(x_{t}\right)<-\varepsilon\left\|x^{m}(t)\right\|^{2}$ holds for any sufficiently small $\varepsilon>0$, which ensure the asymptotic stability of system.

Remark 3. From definition 2, it is clear that

$$
\lim _{t \rightarrow \infty} x(t)=0 \Leftrightarrow \lim _{t \rightarrow \infty} x^{m}(t)=0 .
$$

Remark 4. Considering the norm of $T\left(x^{m-1}(t), x(t-\right.$ $d(t)))$

$$
\begin{aligned}
& \left\|T\left(x^{m-1}(t), x(t-d(t))\right)\right\|_{c}= \\
& \sup _{0 \leq d(t) \leq h}\left\|T\left(x^{m-1}(t), x(t-d(t))\right)\right\| \leq \\
& \sup _{0 \leq d(t) \leq h}\left\|T\left(x^{m-1}(t-d(t)), x(t-d(t))\right)\right\| \leq \\
& \sup _{0 \leq d(t) \leq h}\left\|E x^{m}(t-d(t))\right\|= \\
& \left\|E x^{m}(t-d(t))\right\|_{c}
\end{aligned}
$$

we can get that the inequality of (17) is right.

For system with a constant delay, we can get a delayindependent stability criterion by Theorem 1 .

Corollary 1. For given scalars $h>0$ and $\mu=0$ and a time-varying delay satisfying (3) and (4), the uncertainties satisfy $0 \leq p(t) \leq k$. If there exist $P=P^{\mathrm{T}} \geq 0, Q=Q^{\mathrm{T}} \geq$ 0 , such that

$$
\Phi_{1}=\left(\begin{array}{cc}
\bar{A}_{i}^{\mathrm{T}} P+P \bar{A}_{i}+E^{\mathrm{T}} Q E & P \bar{A}_{d i} \\
* & -Q
\end{array}\right)<0
$$

then the system (1) is asymptotically stable.

\section{Numerical examples}

In this section, we give some examples to illustrate our result. We compare our results with existing works with re- spect to the upper bound of the uncertainties when system is asymptotically stable.

Let us consider the following 2-dimensional time-delay systems with uncertainties

$$
\begin{aligned}
\left(\begin{array}{l}
\dot{x}_{1} \\
\dot{x}_{2}
\end{array}\right)= & \left(\begin{array}{cc}
0 & 1 \\
-2-p(t) & -1
\end{array}\right)\left(\begin{array}{l}
x_{1} \\
x_{2}
\end{array}\right)+ \\
& \left(\begin{array}{cc}
-1 & 0 \\
-1-p(t) & -1
\end{array}\right)\left(\begin{array}{l}
x_{1 d} \\
x_{2 d}
\end{array}\right)
\end{aligned}
$$

with $0 \leq p(t) \leq k$. We can rewrite the system as

$$
\begin{gathered}
\left(\begin{array}{l}
\dot{x}_{1} \\
\dot{x}_{2}
\end{array}\right)=\left[(1-a(t))\left(\begin{array}{cccc}
0 & 1 & -1 & 0 \\
-2 & -1 & -1 & -1
\end{array}\right)+\right. \\
\left.\quad a(t)\left(\begin{array}{cccc}
0 & 1 & -1 & 0 \\
-2-k & -1 & -1-k & -1
\end{array}\right)\right]\left(\begin{array}{c}
x \\
x_{d}
\end{array}\right)
\end{gathered}
$$

with $0 \leq a(t) \leq 1$. We can get $\left[A, A_{d}\right] \in \Omega$.

Assume the delay is time-variant. The upper bound on the delay $h$ is 0.5 and $\mu=0.5$. By calculating the LMI, we can get the the upper bound of uncertainties.

When $m=1$, we can get a feasible solution from [4]. Based on his methods, we can get the upper bound $k=$ 0.76 .

If we choose $m=2$, then $x^{2}=\left[x_{1}^{2}, x_{1} x_{2}, x_{2}^{2}\right]^{\mathrm{T}}$ and $T\left(x, x_{d}\right)=\left[x_{1} x_{d 1}, x_{1} x_{d 2}, x_{2} x_{d 1}, x_{2} x_{d 2}\right]^{\mathrm{T}}$.

We can show that the upper bound $k=0.80$ and the coefficient matrices are given by

$$
\begin{aligned}
F_{1} & =\left(\begin{array}{ccc}
0 & 1 & 0 \\
-2-k & -1 & 0 \\
0 & 0 & 1 \\
0 & -2-k & -1
\end{array}\right) \\
\bar{F}_{d 1} & =\left(\begin{array}{cccc}
-1 & 0 & 0 & 0 \\
-1-k & -1 & 0 & 0 \\
0 & 0 & -1 & 0 \\
0 & 0 & -1-k & -1
\end{array}\right) \\
F_{2} & =\left(\begin{array}{ccc}
0 & 1 & 0 \\
-2 & -1 & 0 \\
0 & 0 & 1 \\
0 & -2 & -1
\end{array}\right), E=\left(\begin{array}{ccc}
1 & 0 & 0 \\
0 & 1 & 0 \\
0 & 1 & 0 \\
0 & 0 & 1
\end{array}\right) \\
\bar{F}_{d 2} & =\left(\begin{array}{cccc}
-1 & 0 & 0 & 0 \\
-1 & -1 & 0 & 0 \\
0 & 0 & -1 & 0 \\
0 & 0 & -1 & -1
\end{array}\right) .
\end{aligned}
$$

When $m=3, x^{3}=\left[x_{1}^{3}, x_{1}^{2} x_{2}, x_{1} x_{2}^{2}, x_{2}^{3}\right]^{\mathrm{T}}$ and $T\left(x^{2}, x_{d}\right)=$ $\left[x_{1}^{2} x_{d 1}, x_{1}^{2} x_{d 2}, x_{1} x_{2} x_{d 1}, x_{1} x_{2} x_{d 2}, x_{2}^{2} x_{d 1}, x_{2}^{2} x_{d 2}\right]^{\mathrm{T}}$.

We can show that the upper bound $k=1.61$ and the 
coefficient matrices are given by

$$
\begin{aligned}
& F_{1}=\left(\begin{array}{cccc}
0 & 1 & 0 & 0 \\
-2-k & -1 & 0 & 0 \\
0 & 0 & 1 & 0 \\
0 & -2-k & -1 & 0 \\
0 & 0 & 0 & 1 \\
0 & 0 & -2-k & -1
\end{array}\right) \\
& \bar{F}_{d 1}=\left(\begin{array}{cccccc}
-1 & 0 & 0 & 0 & 0 & 0 \\
-1-k & -1 & 0 & 0 & 0 & 0 \\
0 & 0 & -1 & 0 & 0 & 0 \\
0 & 0 & -1-k & -1 & 0 & 0 \\
0 & 0 & 0 & 0 & -1 & 0 \\
0 & 0 & 0 & 0 & -1-k & -1
\end{array}\right) \\
& F_{2}=\left(\begin{array}{cccc}
0 & 1 & 0 & 0 \\
-2 & -1 & 0 & 0 \\
0 & 0 & 1 & 0 \\
0 & -2 & -1 & 0 \\
0 & 0 & 0 & 1 \\
0 & 0 & -2 & -1
\end{array}\right), E=\left(\begin{array}{llll}
1 & 0 & 0 & 0 \\
0 & 1 & 0 & 0 \\
0 & 1 & 0 & 0 \\
0 & 0 & 1 & 0 \\
0 & 0 & 1 & 0 \\
0 & 0 & 0 & 1
\end{array}\right) \\
& \bar{F}_{d 2}=\left(\begin{array}{cccccc}
-1 & 0 & 0 & 0 & 0 & 0 \\
-1 & -1 & 0 & 0 & 0 & 0 \\
0 & 0 & -1 & 0 & 0 & 0 \\
0 & 0 & -1 & -1 & 0 & 0 \\
0 & 0 & 0 & 0 & -1 & 0 \\
0 & 0 & 0 & 0 & -1 & -1
\end{array}\right) \text {. }
\end{aligned}
$$

Similarly, we obtain the solutions of $k$ when $m=4$ and $m=5$. Combining $m=1, m=2$ and $m=3$, we can get the Table 1.

Table 1 Allowable upper bound $k$ for various $m$

\begin{tabular}{ccccccc}
\hline$m$ & 1 & 2 & 3 & 4 & 5 & $\cdots$ \\
\hline$k$ & 0.76 & 0.80 & 1.61 & 2.43 & 2.72 & $\cdots$ \\
\hline
\end{tabular}

Table 1 shows the upper bounds on the uncertainties for different $m$ which are obtained from Theorem 1 . It is clear that the homogeneous polynomial LyapunovKrasovskii functions in this paper produce much better results than those from quadratic Lyapunov-Krasovskii functions. In Table 2, although [21] gets $k=2.9$ when $\tau_{m}=0.4$, but $d(t)$ is very conservative at this value. Figs. $1-4$ prove that our results are correct.

Table 2 Allowable upper bound $k$ for [21] with different $\tau_{m}$

\begin{tabular}{cccccccc}
\hline$\tau_{m}$ & 0.01 & 0.05 & 0.1 & 0.15 & 0.2 & 0.3 & 0.4 \\
\hline$k$ & - & - & - & 0.11 & 0.52 & 1.5 & 2.9 \\
\hline
\end{tabular}

Remark 5. In [21], the time-varying continuous function $d(t)$ satisfy $0<\tau_{m} \leq d(t) \leq h$. When $\tau_{m}=0.4$, [21] gets the upper bound of uncertainties $k=2.9(k=2.9>k=$ 2.72 when $m=5$ in this paper). However, the time-varying continuous function $d(t)$ is asked to satisfy $0.4<d(t) \leq 0.5$ at this time. It is obviously that the condition of $d(t)$ is fairly conservative.

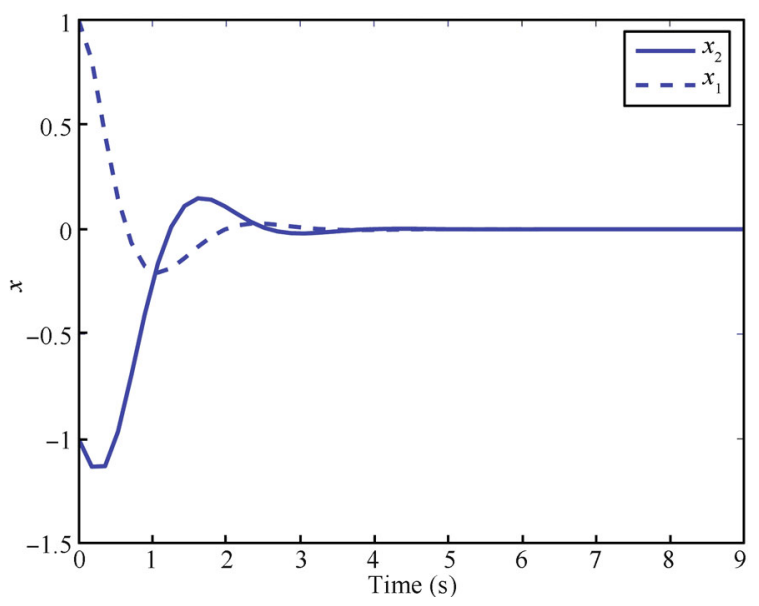

Fig. 1 Responses of state $x$ with $p(t)=0$

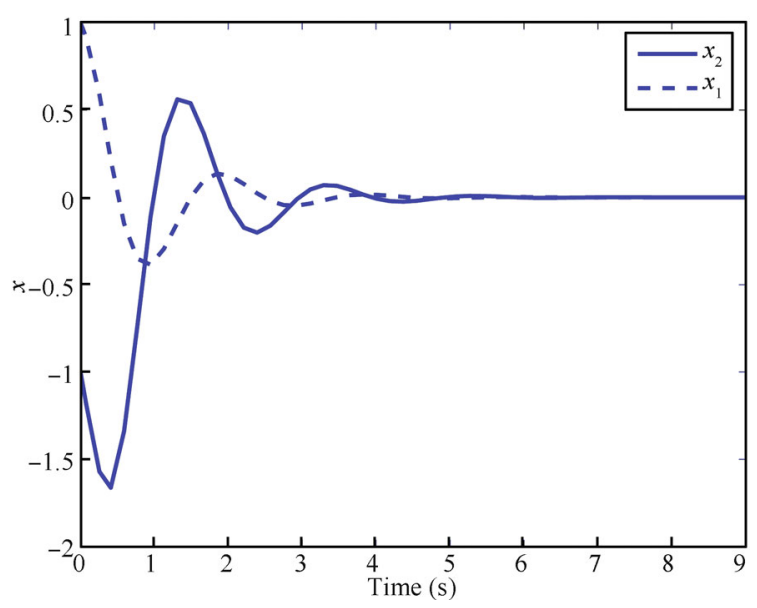

Fig. 2 Responses of state $x$ with $p(t)=1.61$

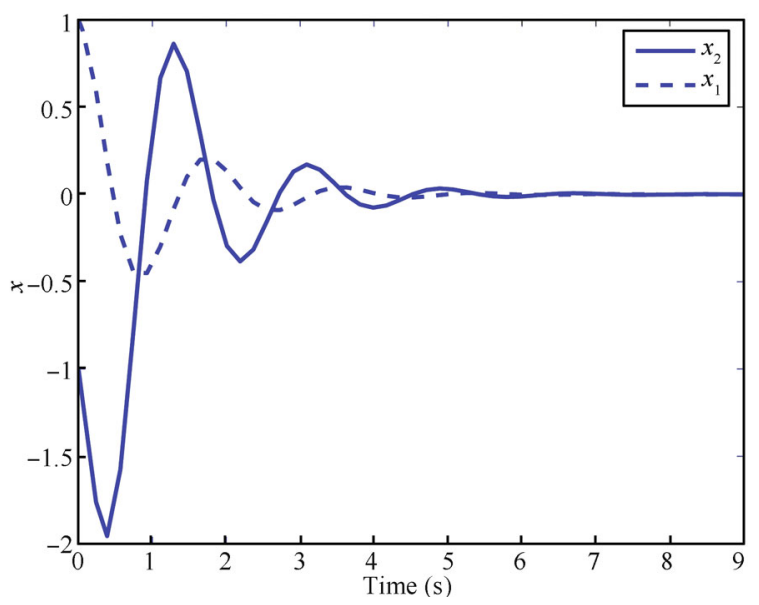

Fig. 3 Responses of state $x$ with $p(t)=2.43$

Remark 6. From Table 1, it is clear that $k$ increases when $m$ increases. However, the bigger $m$ makes calculation more complex. From Figs. 3 and 4, we have the influence of $p(t)=2.43$ and $p(t)=2.72$ for system is similar. So we may consider $k=2.43$ is optimal by considering the complexity of computation. 


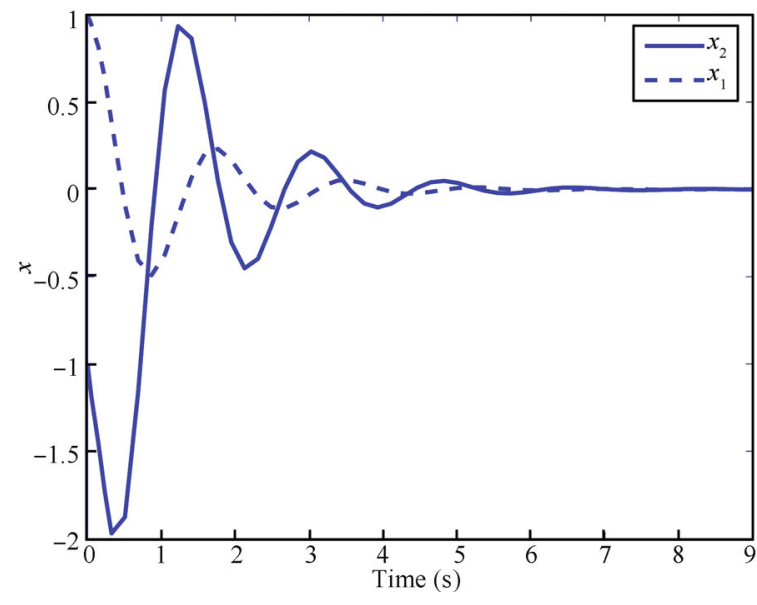

Fig. $4 \quad$ Responses of state $x$ with $p(t)=2.72$

\section{Conclusions}

In this paper, the problem of homogeneous polynomial forms for robustness analysis of uncertain time-delay systems have been investigated. Both delay-independent and delay-dependent criteria are established. Simulation shows the upper bounds on the uncertainties for different $m$ obtained from the theorem. It is clear that the homogeneous polynomial Lyapunov-Krasovskii functions in this paper produce much better results than those by quadratic Lyapunov-Krasovskii functions.

\section{References}

[1] Y. He, M. Wu, J. She. Parameter-dependent Lyapunov functional for stability of time-delay systems with polytopic-type uncertainties. IEEE Transactions on Automatic Control, vol. 49, no. 5, pp. 828-832, 2002.

[2] X. M. Zhang, Q. L. Han. New stability criteria for linear systems with interval time-varying delay. Automatica, vol. 44, no. 10 , pp. 2680-2685, 2008.

[3] S. Boyd, K. Ghaoui, E. Feron, V. Balakrishnan. Linear Matrix Inequalities in Systems and Control Theory, vol. 15, Philadelphia: PA: SIAM, 1994.

[4] M. Wu, Y. He, J. She. Stability Analysis and Robust Control of Time-delay Systems, Beijing, China: Science Press, pp. $43-70,2010$.

[5] E. Fridman, U. Shaked. Delay-dependent stability and $H_{\infty}$ control: Constant and time-varying delays. International Journal of Control, vol. 76, no. 1, pp. 48-60, 2003.

[6] E. Fridman. Stability of systems with uncertain delays: A new "complete" Lyapunov-Krasovskii functional. IEEE Transactions on Automatic Control, vol. 51, no. 5, pp. 885$890,2006$.

[7] J. M. Jiao. A stability criterion for singular systems with two additive time-varying delay components. International
Journal of Automation and Computing, vol.10, no. 1, pp. 39-45, 2013.

[8] Y. S. Lee, Y. S. Moon, W. H, Kwon, P. G. Park. Delaydependent robust $H_{\infty}$ control for uncertain systems with a state-delay. Automatica, vol. 40, no. 1, pp. 65-72, 2004.

[9] Y. Moon, P. Park, W. Kwon, Y. S. Lees. Delay-dependent robust stabilization of uncertain state delayed systems. International Journal of Control, vol.74, no.14, pp.1447$1455,2001$.

[10] X. M. Zhang, Q. L. Han, Novel delay-derivative-dependent stability criteria using new bounding techniques. International Journal of Robust and Nonlinear Control, vol.23, no. 13, pp. 1419-1432, 2013.

[11] Q. L. Han. Improved stability criteria and controller design for linear neutral systems. Automatica, vol.45, no. 8, pp. 1948-1952, 2009.

[12] Z. Zhong, Y. Wu. Globally asymptotic stabilization for nonlinear time-delay systems with ISS inverse dynamics. International Journal of Automation and Computing, vol.9, no. 6, pp. 634-640, 2012.

[13] Z. Jarvis-Wloszek, A. Packard. An LMI method to demonstrate simultaneous stability using non-quadratic polynomial Lyapunov functions. In Proceedings of the 41st IEEE Conference on Decision and Control, IEEE, Las Vegas, USA, pp. 287-292, 2002.

[14] J. Xu, L. H. Xie. Homogeneous polynomial lyapunov functions for piecewise affine systems. In Proceedings of the America Control Conference, IEEE, Portland, USA, pp. 581-586, 2005.

[15] Y. Fujisaki, R. Sakuwa. Estimation of asymptotic stability regions via homogeneous polynomial Lyapunov functions. International Journal of Control, vol. 79, no. 6, pp. 617-623, 2006

[16] G. Chesi, A. Tesi, A. Vicino, R. Genesio. An LMI approach to constrained optimization with homogeneous forms. Systems \& Control Letters, vol. 42, no. 1, pp. 11-19, 2001.

[17] G. Chesi, A. Garulli, A. Tesi, A. Vicino. Homogneous Polynomial Forms for Robustness Analysis of Uncertain Systems, Berlin Germany: Springer-Verlag, pp. 63-97, 2009.

[18] G. Chesi. On the minimum stable commutation time for switching nonlinear systems. IEEE Transactions on Automatic Control, vol. 54, no. 6, pp. 1284-1289, 2009.

[19] G. Chesi, A. Garulli, A. Tesi, A. Vicino. Homogeneous Lyapunov functions for systems with structured uncertainties. Automatica, vol. 39, no. 6, pp. 1027-1035, 2003. 
[20] G. Chesi, Y. S. Hung. Establishing convexity of polynomial Lyapunov functions and their sublevel sets. IEEE Transactions on Automatic Control, vol.53, no. 10, pp. 2431-2436, 2008.

[21] R. Goebel, A. R. Teel, T. Hu, Z. Lin. Conjugate convex Lyapunov functions for dual linear differential inclusions. IEEE Transactions on Automatic Control, vol. 51, no. 4, pp.661666, 2006.

[22] L. Xie, S. Shishkin, M. Y. Fu. Piecewise Lyapunov functions for robust stability of linear time-varying systems. Systems \& Control Letters, vol. 31, no. 3, pp. 165-171, 1997.

[23] A. L. Zelentsovsky. Nonquadratic Lyapunov functions for robust stability analysis of linear uncertain systems. IEEE Transactions on Automatic Control, vol. 39, no. 1, pp. 135138, 1994.

[24] D. Lee, My. Task, Y. Joo. A Lyapunov functional approach to robust stability analysis of continuous-time uncertain linear systems in polytopic domains. International Journal of Control, Automation, and Systems, vol.11, no. 3, pp.460469, 2013.

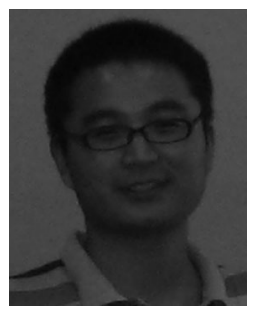

Guo-Chen Pang received the B. Sc. degree from the Department of Mathematics, Lin Yin University, China in 2009 and received his M. Sc. degree from the Institute of Automation, Qufu Normal University, China in 2012. He is currently a Ph. D. candidate at the School of Automation, Southeast University, China.

His research interests include homogeneous polynomial function, domain of attraction and actuator saturation.

E-mail: guochenpang@163.com

ODCID iD: 0000-0003-4974-6308

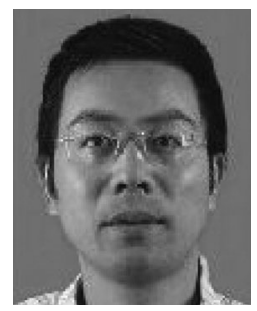

Kan-Jian Zhang received the B. Sc. degree in mathematics from Nankai University, China in 1994, and the M.Sc. and $\mathrm{Ph}$. D. degrees in control theory and control engineering from Southeast University, China in 1997 and 2000. He is currently a professor in Research Institute of Automation, Southeast University, China.

His research is in nonlinear control theory and its applications, with particular interest in robust output feedback design and optimization control.

E-mail: kjzhang@seu.edu.cn (Corresponding author) ORCID iD: 0000-0003-0914-7685 